\title{
Successful treatment of a neonate with persistent vancomycin-resistant enterococcal bacteremia with a daptomycin-containing regimen
}

\author{
Christy A Beneri' \\ David P Nicolau ${ }^{2}$ \\ Howard S Seiden ${ }^{3}$ \\ Lorry G Rubin' \\ 'Pediatric Infectious Diseases, \\ Schneider Children's Hospital - \\ North Shore-LIJ Health System, \\ New Hyde Park, New York, USA; \\ ${ }^{2}$ Center for Anti-Infective Research \\ and Development - Hartford Hospital, \\ Hartford, Connecticut, USA; ${ }^{3}$ Pediatric \\ Cardiology, Schneider Children's \\ Hospital - North Shore-LIJ Health \\ System, New Hyde Park, New York, \\ USA
}

Correspondence: Christy A Beneri Schneider Children's Hospital, North Shore-LIJ Health System, 269-0I 76th Avenue, New Hyde Park, NY I 1040 , USA $\mathrm{Tel}+\mathrm{I} 7184703415$

Fax +I 7184700887

Email cbeneri@nshs.edu

\begin{abstract}
Infections caused by vancomycin-resistant enterococci (VRE) may be difficult to treat because of the limited armamentarium of antimicrobial agents. The difficulty is compounded in pediatric patients in general and neonates in particular because many of the newer antimicrobials have not been studied or approved for children. We report a 3-week-old infant who developed enterococcal bacteremia on post-operative day 10 after a surgical palliation for complex congenital heart disease that was complicated by acute renal failure. Despite removal of vascular catheters and antimicrobial regimens that included linezolid, quinupristin/dalfopristin, ampicillin/sulbactam, rifampin, and gentamicin, bacteremia persisted. It was not cleared until daptomycin (in combination with doxycycline) was started. This is the first case of successful treatment of probable endocarditis due to VRE in a neonate using a daptomycin-containing regimen.
\end{abstract}

Keywords: daptomycin, enterococcus, neonates, pediatrics, endocarditis

\section{Introduction}

Daptomycin is a semisynthetic cyclic lipopeptide antibiotic that is bactericidal in vitro for gram positive organisms including vancomycin-resistant enterococci (Oberholzer et al 2005). Experience with daptomycin in the pediatric population is very limited. We report a case of a neonate with persistent bacteremia caused by vancomycin-resistant enterococcus (VRE) with microbiological cure only after instituting a daptomycincontaining regimen.

\section{Case report}

At 11 days of age a full-term girl with hypoplastic left heart syndrome underwent Stage I Palliation (Norwood with Sano modification) with a $5 \mathrm{~mm}$ Gortex shunt placement. On post-operative day (POD) 10, she developed fever; blood cultures (BACTEC Peds-plus) were taken through a central venous catheter and via venipuncture followed by removal and replacement of vascular catheters. Vancomycin was empirically started (Figure 1). Both blood cultures grew Enterococcus species. Creatinine was $1.6 \mathrm{mg} / \mathrm{dL}$. On POD 13 the blood culture isolates were identified as vancomycin-resistant Enterococcus faecium (VRE, susceptibilities in Table 1) and antimicrobial therapy was changed to linezolid (10 mg/kg/dose every 8 hours). On POD 15 she became afebrile but blood cultures remained positive through POD 18. On POD 16, all vascular catheters were removed and replaced. The antimicrobial was changed to quinupristin/dalfopristin $(7.5 \mathrm{mg} / \mathrm{kg} / \mathrm{dose}$ every 8 hours) on POD 18. Ampicillin-sulbactam (50 mg/kg/dose every 12 hours) was added a day later for possible synergy. The patient remained critically ill. On POD 18 the patient underwent cardiac catheterization; a restricted Sano shunt was found and a stent was placed with improvement. On POD 20 she became febrile. Blood cultures 


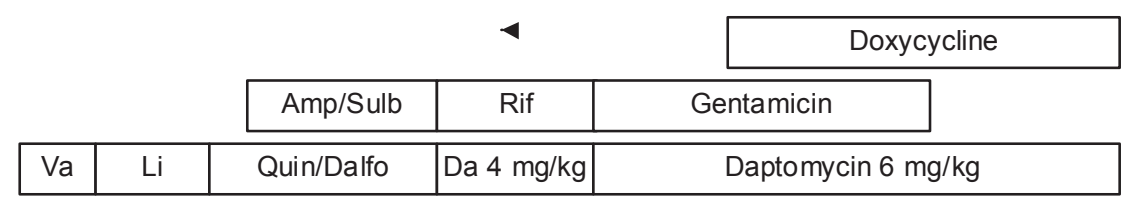

Blood

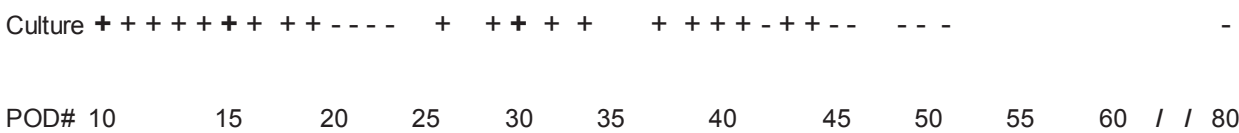

Figure I Patient course in relation to blood culture results and antimicrobial therapy.

Abbreviations: Va,vancomycin; Li, linezolid;Amp/Sulb, ampicillin/sulbactam; Quin/Dalfo,quinprisitin/dalfopristin; Rif, rifampin; Da, daptomycin

obtained on PODs 20, 21, 22, and 23 were negative, but blood cultures obtained on PODs 26, 29, and 30 grew VRE. On POD 30 the antimicrobial was changed to daptomycin at a dose of $4 \mathrm{mg} / \mathrm{kg} /$ dose every 48 hours (serum creatinine, $0.9 \mathrm{mg} / \mathrm{dL}$ ) and rifampin (18 mg/kg/day). Blood cultures obtained on PODs 30, 32, 34, 37, 39-41, 43, and 44 remained positive. On POD 38 rifampin was discontinued and gentamicin was started. One week later doxycycline $(2.5 \mathrm{mg} / \mathrm{kg} /$ dose every 12 hours) was added.

Two weeks into daptomycin therapy the serum creatinine remained elevated to $0.9 \mathrm{mg} / \mathrm{dL}$ and 4 weeks into therapy it was $0.6 \mathrm{mg} / \mathrm{dL}$ where it remained for the duration of treatment. She continued to have positive blood cultures. Five days after starting daptomycin, a trough concentration obtained 24 hours after a dose was $2.77 \mu \mathrm{g} / \mathrm{mL}$, determined using a validated high performance liquid chromatography (HPLC) method at the Center for Anti-Infective Research and Development at Hartford Hospital, and the dosing interval was reduced to 36 hours. The following day a daptomycin peak

Table I Antimicrobial susceptibility for Enterococcus faecium isolate

\begin{tabular}{llll}
\hline Antimicrobial & $\begin{array}{l}\text { Method of } \\
\text { susceptibility Testing }\end{array}$ & $\begin{array}{l}\text { MIC } \\
(\boldsymbol{\mu g} / \mathbf{m L})\end{array}$ & $\begin{array}{l}\text { Susceptibility } \\
\text { interpretation }\end{array}$ \\
\hline Ampicillin & Vitek system (VS)* & $\geq 32$ & $\mathrm{R}$ \\
& E-test & 256 & $\mathrm{R}$ \\
Gentamicin & VS & 500 & $\mathrm{~S}$ \\
Linezolid & VS & 2 & $\mathrm{~S}$ \\
Quinupristin/ & VS & 0.5 & $\mathrm{~S}$ \\
dalfopristin & & & \\
Tetracycline/ & VS & $\mathrm{I}$ & $\mathrm{S}$ \\
doxycycline & & & \\
Vancomycin & VS & $\geq 32$ & $\mathrm{R}$ \\
Daptomycin & E-test & \\
Rifampin & Disk diffusion & I & $\mathrm{S}$ \\
& & applicable & $\mathrm{S}$ \\
\hline
\end{tabular}

Abbreviations: MIC, mean inhibitory concentration. *bioMerieux, Durham, NC; ${ }^{\dagger}$ AB Biodisk, Solona, Sweden. concentration was $6.19 \mu \mathrm{g} / \mathrm{mL}$ and the dose was increased to $6 \mathrm{mg} / \mathrm{kg}$ every 36 hours. Due to continued positive cultures the dosing interval was reduced to 24 hours. Five days later, the peak concentration was $31.32 \mu \mathrm{g} / \mathrm{mL}$. Gentamicin was discontinued 1 week later and doxycycline was continued until end of therapy with daptomycin. It was not until POD 45, after receiving the higher dose of daptomycin for 7 days, that her blood cultures became persistently negative. She received an 8-week daptomycin course including 6 weeks after the last positive blood culture. Creatine phosphokinase (CPK) concentration was normal on daptomycin treatment days 1,8 , and 14 . In follow up the patient did well and tolerated an additional cardiac surgical procedure.

\section{Discussion}

Our patient had persistent VRE bacteremia that was refractory to multiple antimicrobials and removal and replacement of vascular catheters. We presume that endocarditis was present (Saiman 2003; Tissieres et al 2003). Her infection was unlikely vascular catheter-associated given persistence of positive blood cultures despite two changes of the vascular catheter. However, the possibility of septic thrombophlebitis of a large vein could not be completely excluded; no imaging was done specifically to exclude this possibility.

Treatment options for VRE are limited (Torres-Viera et al 2004). Linezolid, approved for use in neonates, and quinupristin/dalfopristin are bacteriostatic for entercocci and were ineffective in our patient. Ampicillin-sulbactam and gentamicin were added for possible synergistic killing (Matsumura et al 1999; Murray 2000). Daptomycin is a new semisynthetic cyclic lipopeptide antibiotic that binds the bacterial cell wall membrane and forms pores, followed by a calcium-dependent action that increases the membrane potential and results in bacterial cell death. A daptomycincontaining regimen was successful in clearing the bacteremia and curing the persistent bloodstream infection. 
Because the patient received short courses of concomitant rifampin and gentamicin and a long course of doxycycline we cannot exclude the possibility that one or more of these antibiotics contributed to the favorable outcome.

Data are limited on the appropriate dosing regimen of daptomycin for pediatric patients. In our patient, clearance of blood cultures correlated with a dose of $6 \mathrm{mg} / \mathrm{kg}$ every 24 hours. However, our patient had impaired renal function (serum creatinine concentration of $0.6 \mathrm{mg} / \mathrm{dL}$; normal, $0.3 \mathrm{mg} / \mathrm{dL}$ ) and daptomycin is excreted through the kidneys. An infant with normal renal function might require more frequent administration. We found 4 published reports of the use of daptomycin in pediatric patients. Adura et al evaluated 16 pediatric patients who received daptomycin at a dose of 4-6 $\mathrm{mg} / \mathrm{kg}$ once daily (except 1 patient with renal dysfunction who received $4 \mathrm{mg} / \mathrm{kg}$ every 48 hours. All but one had infection caused by Staphylococcus aureus and the majority improved with the addition of daptomycin (Adura et al 2007). In a second report, a 13-year-old male status-post bone marrow transplant for aplastic anemia was treated with daptomycin for VRE endocarditis at a dose of $8 \mathrm{mg} / \mathrm{kg}$ once daily (Atkins et al 2006). Study of the pharmacokinetics of daptomycin demonstrated faster clearance than in adults. In a single-dose study, higher clearance rates and shorter halflife of daptomycin was observed in children 2-6 years old compared with children 7-11 or 12-17 years of age or adults (Abdel-Rahman et al 2007). Neonates may require higher and more frequent dosing. Two neonates were treated with $6 \mathrm{mg} / \mathrm{kg} /$ dose every 12 hours (Cohen-Wolkowiez et al 2008). We observed no adverse effects of daptomycin therapy in our patient. Serum CPK was monitored during the first 2 weeks of therapy and not subsequently tested; there was no clinical evidence of rhabdomyolysis. When administered to adults it is recommended that serum CPK be monitored weekly and daptomycin be discontinued if CPK concentration exceeds
5 times the normal concentration (Schriever et al 2005). Successful therapy of our patient with daptomycin suggests this agent may be useful for treatment of pediatric patients with serious infections caused by VRE. Further studies are needed to determine dosing, efficacy, and adverse effects of daptomycin in pediatric patients.

\section{Disclosures}

David Nicolau receives Grant support from Cubist Pharmaceuticals and he is on the Speaker's Bureau for the company.

\section{References}

Adura MI, Mejias A, Katz KS, et al. 2007. Daptomycin therapy for invasive gram-positive bacterial infections in children. Pediatr Infect Dis J, 26:1128-32.

Atkins, RL, Haase MR, Levy EN. 2006. Pharmacokinetics of daptomycin in a critically ill adolescent with vancomycin-resistant enterococcal endocarditis. Pharmacotherapy, 26:694-8.

Abdel-Rahman SM, Benziger DP, Jacobs RF, et al. 2008. Single-dose pharmacokinetics of daptomycin in children with suspected or proven gram-positive infections. Pediatr Infect Dis J, 27:330-4.

Cohen-Wolkowiez M, Smith PB, Benjamin DK, et al. 2008. Daptomycin use in infants: report of two cases with peak and trough drug concentrations. Journal of Perinatology, 28:233-4.

Murray BE. 2000. Vancomycin-resistant enterococcal infections. $N$ Engl $J$ Med, 342:710-21.

Matsumura SO, Louie L, Louie M, et al. 1999. Synergy testing of Vancomycin-resistant Enterococcus faecium against quinupristin-dalfopristin in combinations with other antimicrobial agents. Augment Altern Commun, 43:2776-9.

Oberholzer CA, Caserta MT. 2005. Antimicrobial update: daptomycin. Pediatr Infect Dis J, 24:919-20.

Saiman L. 2003. Endocarditis and intravascular infections. In: Long S, Pickering L, Prober C (eds). Principles and Practices of Pediatric Infectious Diseases. 2nd ed. Philadelphia: Churchill Livingston. p 253.

Schriever CA, Fernandez KA, Rodvold KA, et al. 2005. Daptomycin: a novel cyclic lipopeptide antimicrobial. Am J Health Syst Pharm, 62:1145-58.

Tissieres P, Gervaix A, Beghetti M, et al. 2003. Value and limitations of the vonReyn, Duke, and modified Duke criteria for the diagnosis of endocarditis in children. Pediatrics, 112:467-71.

Torres-Viera C and Dembry L-M. 2004. Approaches to vancomycin-resistant enterococci. Curr Opin Infect Dis, 17:541-7. 
